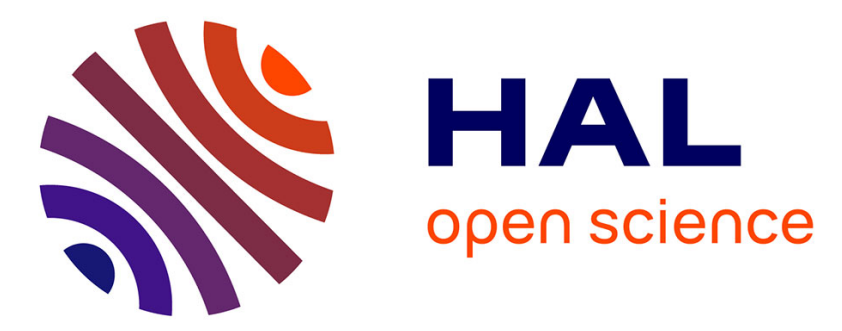

\title{
Minimizing hallucination in Histogram of Oriented Gradients
}

\author{
Javier Ortiz, Slawomir Bak, Michal Koperski, François Brémond
}

\section{To cite this version:}

Javier Ortiz, Slawomir Bak, Michal Koperski, François Brémond. Minimizing hallucination in Histogram of Oriented Gradients. The 12th IEEE International Conference on Advanced Video and Signal-based, Aug 2015, Karlsruhe, Germany. hal-01199386

\section{HAL Id: hal-01199386 \\ https://hal.inria.fr/hal-01199386}

Submitted on 15 Sep 2015

HAL is a multi-disciplinary open access archive for the deposit and dissemination of scientific research documents, whether they are published or not. The documents may come from teaching and research institutions in France or abroad, or from public or private research centers.
L'archive ouverte pluridisciplinaire HAL, est destinée au dépôt et à la diffusion de documents scientifiques de niveau recherche, publiés ou non, émanant des établissements d'enseignement et de recherche français ou étrangers, des laboratoires publics ou privés. 


\title{
Minimizing hallucination in Histogram of Oriented Gradients
}

\author{
Javier Ortiz Sławomir Bąk Michał Koperski François Brémond \\ INRIA Sophia Antipolis, STARS group \\ 2004, route des Lucioles, BP93 \\ 06902 Sophia Antipolis Cedex - France \\ firstname.surnamedinria.fr
}

\begin{abstract}
Histogram of Oriented Gradients is one of the most extensively used image descriptors in computer vision. It has successfully been applied to various vision tasks such as localization, classification and recognition. As it mainly captures gradient strengths in an image, it is sensitive to local variations in illumination and contrast. In the result, a normalization of this descriptor turns out to be essential for good performance [3, 4]. Although different normalization schemes have been investigated, all of them usually employ L1 or L2-norm. In this paper we show that an incautious application of L-like norms to the HOG descriptor might produce a hallucination effect. To overcome this issue, we propose a new normalization scheme that effectively minimizes hallucinations. This scheme is built upon a detailed analysis of the gradient distribution resulting in adding an extra bin with a specific value that increases HOG distinctiveness. We validated our approach on person re-identification and action recognition, demonstrating significant boost in the performance.
\end{abstract}

\section{Introduction}

Histogram of Oriented Gradients (HOG) is one of the most popular descriptors for characterizing image regions. In [3], authors cover the importance of the normalization step when using HOG since the descriptor is sensitive to illumination and contrast changes. They claim that without a proper normalization, the performance of their human detection framework drops by $27 \%$.

Although many different normalization schemes have been investigated, the core of all of them is L1 or L2-norm. In this paper we show that an incautious application of Llike norms to the HOG descriptor might produce a hallucination effect, i.e. specific properties of high texture can appear while describing a texture-less image. An example of this can be seen in figure 1. It illustrates histogram

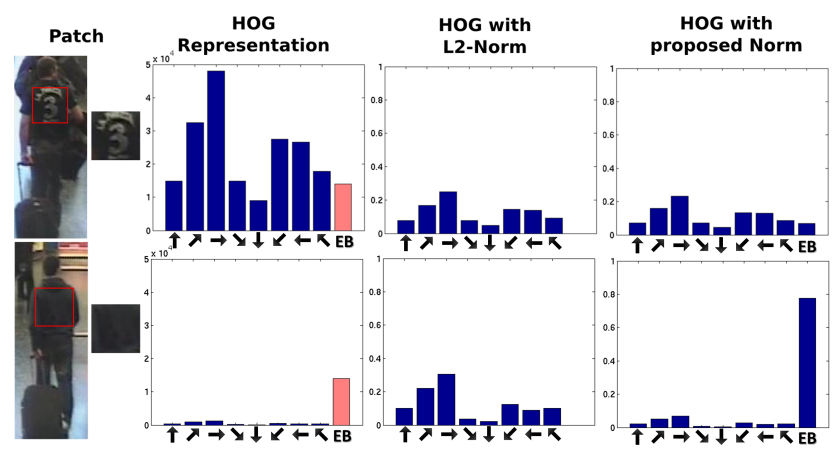

Figure 1: HOG representations of two patches with different amount of texture. Each bin represents the sum of edge magnitudes in a specific orientation. In the third column it can be seen that after L2-Norm both representations became very similar while they remained different in the fourth column where the proposed bin marked as EB was added.

representations of two significantly different image regions: high texture (top row) and low texture (bottom row). From the second column (HOG REPRESENTATION) it is clear that there is a big difference between HOG representations (in blue color) because the number of edges in the texture-less patch is at least one order of magnitude lower. However, after L2-normalization, this difference disappears making both image regions indistinguishable. We call this undesired effect as hallucination because some elements (in this case edges) appear in the HOG representation while they do not actually exist in the original image. As a result, two image regions can have similar final HOG representation while being actually very different. This might decrease the accuracy in any recognition system based on the HOG descriptor.

To overcome this issue, we propose to add an extra bin (EB) with a specific value to the HOG histogram before a normalization step. This bin is illustrated in red in figure 1. We claim that the EB helps to differentiate between patches that contain texture from the ones that do not. Note that the histograms in the last column maintain the difference 
after the L2 normalization. The representation for the textured patch does not change considerably while in the nontextured patch, the extra bin becomes significantly higher compared to the rest of the bins.

Normalization with EB adds two types of information for differentiating between textured and non-textured patches: (1) it helps to maintain the scale of bins when their value is low; (2) after the normalization EB contains information on the scale of the histogram; it gets higher for non-textured patches and lower for textured ones. We discovered that the value of EB can have a significant impact on the performance w.r.t. the recognition task.

This paper makes the following contributions:

- We propose to add the extra bin (EB) before applying any histogram normalization. This keeps HOG descriptor highly discriminative.

- We perform a detailed analysis of EB w.r.t. its value and the descriptor performance on different vision tasks. We offer a learning scheme of the EB value. Our learning is based on modelling an integral of gradient as a Mixture of two Gaussian distributions. We found that the optimal value of EB corresponds to the intersection of these distributions.

- We validated our method on 3 datasets for person reidentification and 2 datasets for action recognition obtaining surprising results.

This paper is organized as follows. Related work on HOG descriptor and its normalization schemes are covered in section 2. Contributions are described in section 3. Finally, we validate our approach in section 4 and conclude in section 5 .

\section{Related work}

Histogram of Oriented Gradients (HOG) is one of the most popular representations for image regions due to its successful application to various vision tasks such as object detection, classification and recognition. Introduced by [3] for detecting pedestrians, it significantly outperformed existing state of the art detectors. Given an image, it extracts intensity (or color) gradient for each pixel using finite difference filters $\left([-1,0,+1],[-1,0,+1]^{t}\right)$ computing pixel orientation and magnitude. Pixel magnitudes are grouped w.r.t. their discretized orientations producing histograms. Already [3] illustrates that such representation is sensitive to local variations in illumination and contrast, making a normalization step crucial for good performance. Authors use four different normalization factors for obtaining a robust representation. These factors are extracted from surrounding image regions computed as L2-norms. The final feature vector is a concatenation of given normalization responses.
Same representation is employed in [14] for identity recognition.

In [4] on top of this feature vector, principal component analysis is applied for reducing dimensionality. By detailed analysis of eigenvectors, [4] proposes analytic dimensionality reduction without loss of the performance. This analytic reduction is also involving L2-norm.

Action recognition is other vision domain that extensively employs HOG descriptor for differentiating appearance. Although in this domain motion descriptors (e.g. $\mathrm{HOF}$ [9], MBH[16]) are the most useful for recognizing actions, [16] shows that appearance based descriptors carry complementary information and can boost performance. HOG descriptor is one of the most popular appearance descriptor used together with local spatio-temporal feature methods to encode local appearance. Such combination of motion and appearance based descriptor helps to distinguish actions with similar motion pattern but different appearance (e.g. actions involving different objects). All these methods usually employ L2-norm before the learning step.

The issue related to the previously mentioned hallucination effect has recently been mentioned in OTC descriptor [11] for scene categorization problem. Authors came up with the idea of adding a fixed small-value bin (0.05) to the histogram before applying L2-norm. We claim that the value of this additional histogram has significant impact on the performance (see section 4). As a result, instead of having a fixed-value bin, we propose to learn this value based on a gradient distribution computed from a random sampling of the given dataset. Assuming Gaussian distributions of textured and texture-less image regions we employ Expectation Maximization algorithm for finding the optimal bin value. Demonstrated experiments on diverse vision tasks and datasets confirm our claims.

\section{Approach Overview}

\subsection{Histogram of Oriented Gradients}

As it was explained in previous sections, each HOG bin represents the magnitude of edges that a patch contains in a specific range of orientations. In cases where the image has size or illumination changes, the final representation of the same person in two cameras could be very different. Using histogram normalization in HOG, this variations can be attenuated because this step sets the same range of values for each representation. Examples of HOG representations can be seen in fig. 1 where a patch is extracted from an image and then each bin in the histogram represents the strength of edges in each orientation. Applying L2-norm scheme, the hallucination problem can be seen in the third column where the differences between histograms are very low while they come from two very different images.

We propose a new normalization scheme where an extra 
bin is added to HOG representations before normalization step. This bin has a specific value that should be found in order to have optimal performance. This step is explained below while the entire algorithm is detailed in subsection 3.4.

\subsection{Distribution of the sum of bins}

The first step to find the optimal value for the extra bin is to calculate the HOG representation from a considerable number of random patches in the dataset. In this case, each bin represents the sum of magnitudes for all pixels in the patch grouped w.r.t. their orientations.

With this information, the following step is to calculate the sum for all bins in each HOG descriptor and plot them in a histogram that shows the frequency of appearance for each sum of values in this particular dataset. In other words, having bin $b_{i}$ that represents a particular orientation in a HOG descriptor and number of bins $n$ for each histogram, we express the sum of all bins of a particular patch $j$ as:

$$
s_{j}=\sum_{i=1}^{n} b_{i}
$$

Following this idea, we sample patches from i-LIDSMA[1] (person re-identification dataset) and we plot $s_{j}$ values w.r.t. their frequency, generating distribution $S=p(s)$ (in blue in fig. 2).

\subsection{Expectation Maximization}

From figure 2 it is clear that distribution $S$ is a mixture of distributions. Thus, we model $S$ as a mixture of two Gaussian distributions:

$$
S=\mathcal{N}_{1}\left(\mu_{1}, \sigma_{1}\right)+\mathcal{N}_{2}\left(\mu_{2}, \sigma_{2}\right) .
$$

We believe that the left side of $S$ corresponds to texture-less patches while the right side corresponds to textured patches. We applied Expectation Maximization (EM) algorithm to find the parameters $\left(\mu_{1}, \mu_{2}, \sigma_{1}, \sigma_{2}\right)$ of $\mathcal{N}_{1}$ and $\mathcal{N}_{2}$ that stand for a Gaussian Mixture Model (GMM).

Following the experiments described in subsection 4.1 we perform re-identification tests setting different values for EB and we get the results shown in fig. 2 (red curve) for i-LIDS-MA where the highest re-identification rate is obtained for $\mathrm{EB}=s_{x}$. Note the red y-axis on the right side illustrating the recognition performance w.r.t. value of EB.

It can also be seen in fig. 2 that the value of $s_{x}$ obtained experimentally corresponds to the value where two Gaussian distributions intersect. This relation means that EB value should be chosen in the intersection between the two modelled Gaussians in order to keep as much texture-less patches in the left and textured patches in the right.

Once the intersection is found, we use it to set the EB value in HOG descriptor for all the patches in the dataset. Finally, we normalize descriptors using L2-norm and continue with the normal work flow w.r.t. the given application.

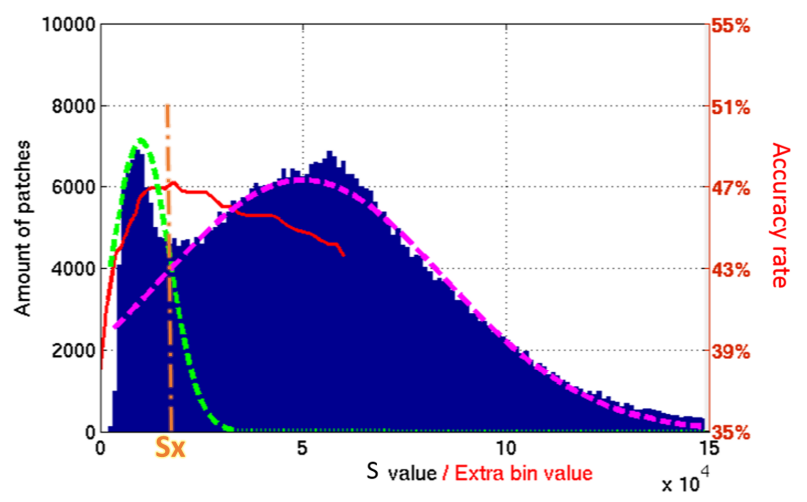

Figure 2: In blue: histogram of $S$ (sum of bins) for HOG descriptors in i-LIDS-MA dataset. Red curve shows the first rank recognition rate w.r.t. to different EB - see the y-axis on the right side). Green and purple curves illustrate two Gaussian distributions obtained by employing Expectation Maximization. Peak of performance can be seen for $\mathrm{EB}=$ $s_{x}$, where two distribution intersect.

\subsection{Algorithm}

As a summary, we present the steps of our algorithm:

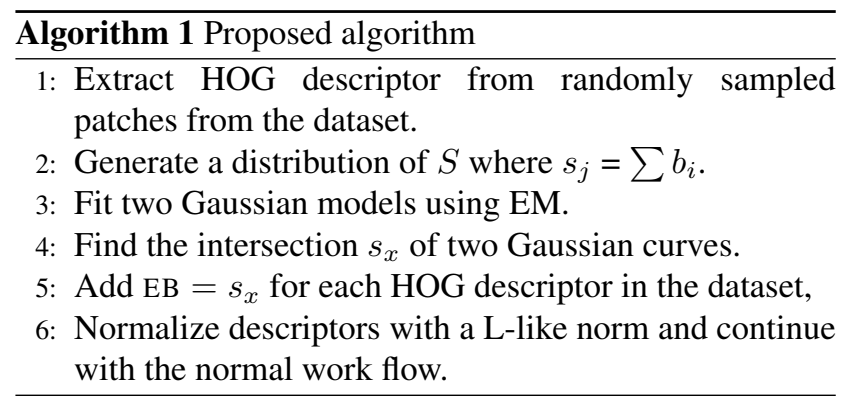

\section{Experiments}

\subsection{Person re-identification}

During the past few years person re-identification has been the focus of intense research bringing new metrics and datasets for evaluation. We carry out our experiments on three challenging datasets: i-LIDS-MA[1], i-LIDS-AA[1] and PRID 2011[6].

In our experiments, we follow a single-shot scheme that uses only a randomly selected single image for generating gallery and probe signatures.

Every cropped pedestrian image is scaled into a fixed size window of $64 \times 192$ pixels. A set of rectangular subregions is produced by shifting $32 \times 32$ regions with a 16 pixels step. This operation results in 33 overlapping rectangular sub-regions. From each sub-region, we extract a HOG descriptor consisting of 8 bins. These descriptors are concatenated in order to form the subject signature. 

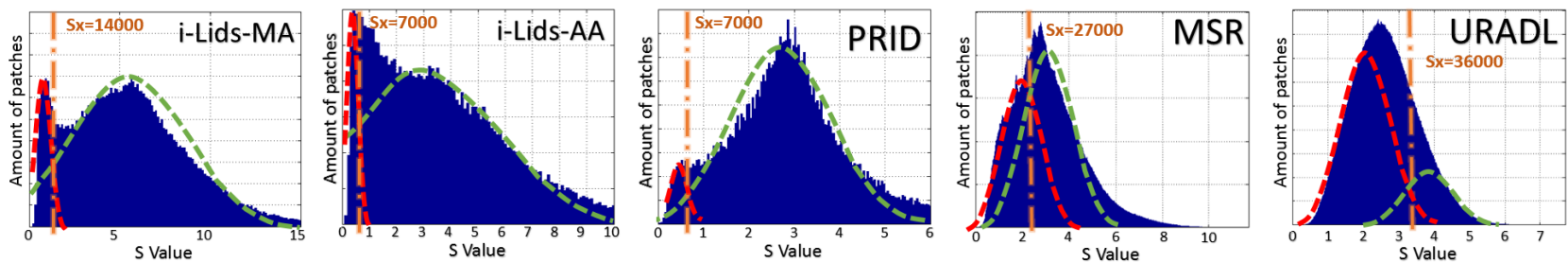

Figure 3: Distribution of the sum of bins for randomly sampled patches for each dataset (scale on $\mathrm{x}$-axis is $\times 10^{4}$ ). Modelled distributions are drawn in red and green.

In the evaluation step, each signature is used as a query to the gallery set of signatures from different cameras. We repeat experiments 20 times and then average obtained results. Algorithms performance are analysed in terms of recognition rate for the first rank and using the cumulative matching characteristic (CMC) curve [5]. This curve represents the expectation of finding the correct match in the top $n$ matches. Note that in all experiments we use Euclidean distances for comparing signatures. On top of this framework we can easily apply any metric learning approach. Metric learning performance w.r.t. EB is beyond the scope of this paper.

i-LIDS-MA[1]: This datasets have been extracted from the 2008 i-LIDS Multiple-Camera Tracking Scenario (MCTS) dataset with multiple non-overlapping camera views. It consists of 40 individuals manually selected. For each individual a set of 46 images is given. Hence, we have in total $40 \times 2 \times 46=3680$ images.

Table 1 and figure 4 shows that using our approach, we can obtain $+9.2 \%$ of improvement compared to not using EB. As it can be seen in fig. 3, i-LIDS-MA presents the best distinguishable Gaussian distributions. This means that the dataset contains a well defined difference between patches with and without texture and therefore the use of the proposed EB has significant impact on the performance. We also report the HOG performance w.r.t. to different EB values.

Table 1 also illustrates a comparison with state of the art results on this dataset. Our approach, although very simple, outperforms both OMRR and RDC methods. We do not outperform CML but it is important to note that in experiments we use just a simple Euclidean distance instead of employing Metric Learning. The main reason of such choice is to have fully unsupervised framework for re-identification.

i-LIDS-AA[1]: This dataset contains 100 individuals automatically detected and tracked in two cameras. Cropped images are noisy, which makes the dataset more challenging (e.g. detected bounding boxes are not accurately centred around the people, only part of the people is detected due to
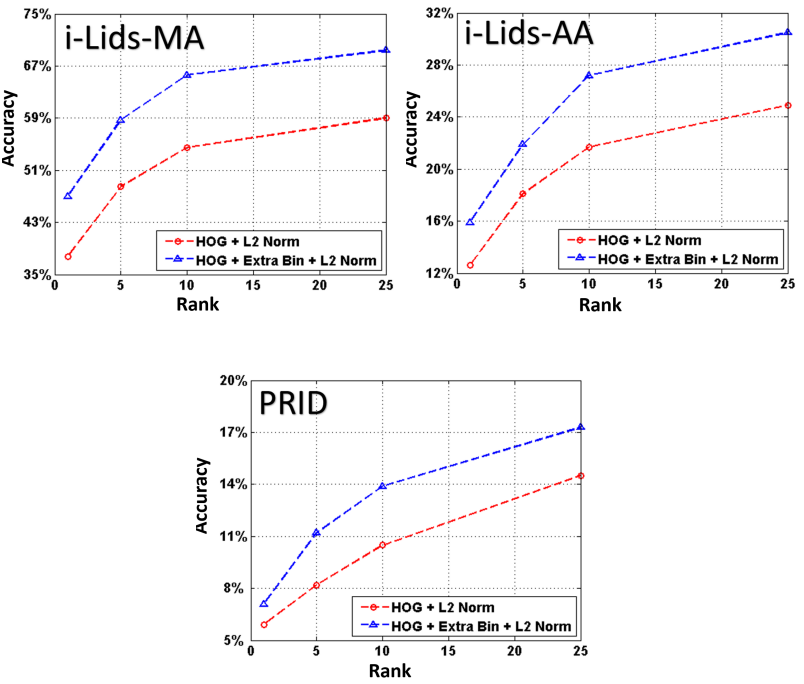

Figure 4: Performance comparison with and with out using our approach on Person Re-identification datasets.

occlusion).

The performance of EB using our algorithm is also reported in Table 1 and fig. 4 illustrates a comparison of the HOG descriptor performance with and without the proposed EB. In this case, our approach is outperformed by Metric Learning based algorithms.

The performance improvement is less than in i-LIDSMA because in this dataset, both distributions are very close between each other so, in consequence, there are a lot of miss-predictions when trying to set a value that differentiate non-textured from textured patches (see fig. 3).

PRID 2011 dataset[6]: This is a multi-shot dataset that consists of images extracted from multiple person trajectories with two static cameras. The dataset contains images from 475 person trajectories from one camera and 753 from the other one with 245 persons appearing in both views. Images present viewpoint, illumination and background changes between cameras but also people are captured with different poses since images are extracted from trajectories. Finally, this dataset contains 5 images per trajectory. 


\begin{tabular}{|l|c|c|c|}
\hline Method/Dataset & i-LIDS-MA & i-LIDS-AA & PRID2011 \\
\hline Standard HOG & $37.8 \%$ & $12.6 \%$ & $5.9 \%$ \\
\hline CML[10] & $\mathbf{4 8 . 5 \%}$ & $\mathbf{1 9 . 4 \%}$ & - \\
OMRR[10] & $45.8 \%$ & $18.5 \%$ & - \\
RDC[10] & $44.0 \%$ & $16.7 \%$ & - \\
HIRZER 2011[6] & - & - & $4.0 \%$ \\
HIRZER 2012[7] & - & - & $\mathbf{1 5 . 0 \%}$ \\
\hline \multicolumn{4}{|c|}{ Our Method } \\
\hline EB=5000 & $43.0 \%$ & $15.7 \%$ & $7.0 \%$ \\
EB $=10000$ & $45.5 \%$ & $15.4 \%$ & $6.2 \%$ \\
EB $=20000$ & $45.4 \%$ & $15.2 \%$ & $6.3 \%$ \\
EB $=s_{x}$ & $47.0 \%$ & $15.9 \%$ & $7.1 \%$ \\
\hline
\end{tabular}

ities of Daily Living dataset contains 10 types of human activities of daily living. In total the dataset contains 150 video sequences. Actions in the data-set are difficult to classify based on a single descriptor (e.g. eating a banana vs. eating snack chips). Thus appearance based descriptor like HOG provides important information, as addition to motion based descriptors.

We use leave-one-person-out cross-validation evaluation scheme. In table 2 we report performance of proposed method with different EB values compared to standard HOG. When we use EB based on proposed Gaussian intersection method, we report a gain in the performance, comparing to standard HOG. However, our results also

Table 1. Accuracy results for first rank on Person Re-identification datasets. Values of $s_{x}$ are 14000, 7000 and 7000 respectively (see fig. 3)

In fig 3, PRID 2011 presents a distribution where it can be seen that the amount of non-textured patches is very low compared to textured patches. This situation made that the improvement of our approach is much lower than in other datasets. Table 1 shows that while our approach performs better than without using EB, it is still worse than other approaches. This can be explained as we are not using any Metric Learning approach in our framework like in [7].

\subsection{Action Recognition}

In this section, we present the evaluation of our approach using two state-of-the-art datasets: URADL [12] and MSRDailyActivity3D [18]. We study the performance of HOG appearance descriptor with and without extra bin. The standard HOG descriptor is the most popular appearance descriptor for action recognition, and therefore, it provides a good baseline for comparison. In many works [18, 9] authors combine appearance descriptors with motion-based descriptors. We also combine proposed method with Dense Trajectories motion features (trajectory shape, HOF, MBH).

For evaluation we extract local spatio-temporal patches from a video sequence. For this task we use Dense Trajectories detector, but it is worth mentioning that any other detector can be used. We follow the same procedure as in [16] and we cut the patch around each trajectory point. Then for each patch we compute HOG descriptor with standard normalization and with proposed extra bin. Finally we follow standard Fisher Vectors [15] framework, where we encode a video sequence using first and second order statistics of a distribution of a descriptor feature set $\mathbb{X}$. Based on such representation we train linear SVM. To fuse together different descriptors we simply concatenate Fisher Vector representation before training SVM.

URADL dataset[12]: The University of Rochester Activshow that we can find a better EB value by exhaustive search on validation-set. We found that relatively big EB values improve HOG performance. This situation can be explained that extracted patches were selected using Dense Trajectories detector - therefore many patches are highly textured. It is visible in figure 3 , where distribution for URADL does not have clear separation between two Gaussians. In addition when we select relatively big EB value, it means that we focus more on the fact that EB after normalization contains information on the scale of the histogram (see section 1). Moreover, big values of EB suppress small differences in HOG descriptors (patch details). In the result, two patches that share common pattern but differ in details, after adding EB with a high value become more similar. Such property is desired in action recognition, as very often we look for the rough appearance correspondence of objects involved in the action, rather than exact match. In table 3 we report performance of fusion with motion based descriptors where our approach reaches state-of-the-art results.

MSRDailyAcivity dataset[18]: This dataset consists of 16 actions performed by 10 subjects. In addition each action is performed in standing and sitting position which adds an additional intra-class variation.

We use leave-one-person-out cross-validation evaluation scheme. In table 2 we report performance of different EB values compared to standard HOG. In this dataset, we have the same situation as with URADL dataset. But the differences between performance of different EB values are smaller than in URADL. This fact can be explained that in MSRDailyActivity3D actors are farther from camera comparing to URADL, thus appearance of objects involved in action is smaller and HOG descriptor contributes less to overall accuracy. In table 4 we report performance of fusion with motion based descriptors. Our method is outperformed only by methods which use skeleton, which requires additional segmentation step and depth information from sensor. However our method achieves competitive results using only RGB video sequence. 


\begin{tabular}{|l|r|r|}
\hline Method/Dataset & URADL & $\begin{array}{r}\text { MSRDaily } \\
\text { Activity3D }\end{array}$ \\
\hline Standard HOG & $82.67 \%$ & $55.31 \%$ \\
\hline \multicolumn{3}{|c|}{ Our Method } \\
\hline EB $=100000$ & $87.33 \%$ & $56.25 \%$ \\
EB $=40000$ & $86.00 \%$ & $58.44 \%$ \\
EB $=60000$ & $\mathbf{9 0 . 6 7 \%}$ & $\mathbf{5 8 . 7 5 \%}$ \\
EB $=6000$ & $46.97 \%$ & $55.31 \%$ \\
EB $=s_{x}$ & $85.67 \%$ & $58.44 \%$ \\
\hline
\end{tabular}

Table 2. Results on Action Recognition datasets. The $s_{x}$ values are 27000 and 36000 respectively.

\begin{tabular}{|l|r|}
\hline Approach & Accuracy \\
\hline Messing[12] & $89.00 \%$ \\
Bilinski[2] & $92.00 \%$ \\
Wang[17] & $\mathbf{9 6 . 0 0 \%}$ \\
DT - DenseTrajectories & $93.33 \%$ \\
DT - with our method & $94.00 \%$ \\
\hline
\end{tabular}

Table 3. Results of fusion with motion model on URADL dataset.

\begin{tabular}{|l|r|}
\hline Approach & Accuracy \\
\hline Dynamic Time Warping[18] & $54.00 \%$ \\
HON4D[13] & $80.00 \%$ \\
Actione Ensemble[18] & $85.00 \%$ \\
3D Trajectories[8] & $72.00 \%$ \\
DT - DenseTrajectories & $77.81 \%$ \\
DT - with our method & $\mathbf{8 0 . 3 1 \%}$ \\
\hline
\end{tabular}

Table 4. Results of fusion with motion model on MSRDailyActivity3D dataset. Please note that approaches in italic requires skeleton detection and depth information.

\section{Conclusion}

We proposed the new normalization scheme of the HOG descriptor that effectively minimizes a hallucination effect. We modeled the integral of gradient as the Mixture of two Gaussian distributions obtaining a separation of high and low texture images. The intersection of these distribution gives us the value of the extra bin (EB) that is added the set of gradient orientations in the HOG descriptor. We demonstrated that while normalizing HOGs, the value of EB has significant impact on the performance. We showed that our approach consistently improves recognition rate in both person re-identification and action recognition. The proposed EB gave optimal results for person re-identification while performing sub-optimally for action recognition. Further analysis will focus on searching optimal EB for action recognition and on extending re-identification by metric learning approaches.

\section{References}

[1] S. Bak, E. Corvee, F. Bremond, and M. Thonnat. Boosted human re-identification using Riemannian manifolds. IMAVIS, 2011. 3, 4

[2] P. Bilinski, E. Corvee, S. Bak, and F. Bremond. Relative dense tracklets for human action recognition. In $F G, 2013.6$

[3] N. Dalal and B. Triggs. Histograms of oriented gradients for human detection. In CVPR, 2005. 1, 2

[4] P. F. Felzenszwalb, R. B. Girshick, D. McAllester, and D. Ramanan. Object detection with discriminatively trained part based models. TPAMI, 2010. 1, 2

[5] D. Gray, S. Brennan, and H. Tao. Evaluating appearance models for recognition, reacquisition, and tracking. In PETS, 2007. 4

[6] M. Hirzer, C. Beleznai, P. M. Roth, and H. Bischof. Person re-identification by descriptive and discriminative classification. In SCIA, 2011. 3, 4, 5

[7] M. Hirzer, P. M. Roth, and H. Bischof. Person reidentification by efficient impostor-based metric learning. In AVSS, 2012. 5

[8] M. Koperski, P. Bilinski, and F. Bremond. 3D Trajectories for Action Recognition. In ICIP, 2014. 6

[9] I. Laptev, M. Marszalek, C. Schmid, and B. Rozenfeld. Learning realistic human actions from movies. In $C V P R, 2008.2,5$

[10] W. Li, Y. Wu, M. Mukunoki, and M. Minoh. Coupled metric learning for single-shot versus single-shot person reidentification. Optical Engineering, 2013. 5

[11] R. Margolin, L. Zelnik-Manor, and A. Tal. Otc: A novel local descriptor for scene classification. In ECCV, 2014. 2

[12] R. Messing, C. Pal, and H. Kautz. Activity recognition using the velocity histories of tracked keypoints. In ICCV , 2009. 5, 6

[13] O. Oreifej and Z. Liu. Hon4d: Histogram of oriented $4 \mathrm{~d}$ normals for activity recognition from depth sequences. In $C V P R, 2013.6$

[14] O. Oreifej, R. Mehran, and M. Shah. Human identity recognition in aerial images. In $C V P R, 2010.2$

[15] F. Perronnin, Y. Liu, J. Sanchez, and H. Poirier. LargeScale Image Retrieval with Compressed Fisher Vectors. In CVPR, 2010. 5

[16] H. Wang, A. Klaser, C. Schmid, and C.-L. Liu. Action recognition by dense trajectories. In $C V P R, 2011$. 2, 5

[17] J. Wang, Z. Chen, and Y. Wu. Action recognition with multiscale spatio-temporal contexts. In CVPR, 2011. 6

[18] Y. Wu. Mining actionlet ensemble for action recognition with depth cameras. In $C V P R, 2012.5,6$ 